\title{
Complex-Coefficient Microwave Photonic Filter Based on Orthogonally Polarized Optical Single-Sideband Modulation
}

\author{
Jinwang QIAN ${ }^{\mathrm{a}}$, Junling SUN ${ }^{\mathrm{a}}$, Pengge MA ${ }^{\mathrm{a},{ }^{*}}$, Xinlu GAO ${ }^{\mathrm{b}}$ and Shanguo HUANG ${ }^{\mathrm{b}}$ \\ ${ }^{a}$ Zhengzhou University of Aeronautics, Zhengzhou 450046, China \\ ${ }^{b}$ Beijing University of Posts and Telecommunications, Beijing 100876, China
}

\begin{abstract}
A complex-coefficient microwave photonic filter with continuous tunability is proposed and experimentally demonstrated. The filter taps are based on a $360^{\circ}$ tunable microwave photonic phase shifter, which is realized by orthogonally polarized optical single-sideband (OSSB) modulation. The experimental results are shown and regarded as good performance for the proposed filter. The phase shift for the two taps covers a full $360^{\circ}$ range from $8 \mathrm{GHz}$ to 26 $\mathrm{GHz}$. Frequency responses with different center frequency are measured within $20-21 \mathrm{GHz}$ with the full free spectral ranges (FSRs) of $185 \mathrm{MHz}$ and $285 \mathrm{MHz}$, respectively.
\end{abstract}

Keywords. Microwave photonic filter, complex coefficient, microwave photonic phase shifter

\section{Introduction}

Microwave photonic filter (MPF) has important applications in the processing of microwave signals due to its inherent advantages, including broad bandwidth, high tunability, and immunity to electromagnet interference [1]. Complex-coefficient filters are able to keep the FSR and shape unchanged while tuning the center frequency, so they have the most potential for applications among the MPFs with different coefficients. Many MPFs with complex coefficients have been reported [2-6] including those based on SSB modulation [2], stimulated Brillouin scattering (SBS) [3], fiber Bragg grating (FBG) [4], programmable photonic processor [5]. However, the problem of bias drift is existed in the scheme based on SSB modulator. The structure of MPF based on SBS is complex since it needs two or more modulators. The key limitation associated with FBG-based method is the limited tuning range. Furthermore, these schemes utilize two or more laser sources, making the system costly and complicated. In [6], a complex-coefficient MPF using one single optical source is proposed. However, the phase difference between the orthogonally polarized signals should be maintained at $\pi / 2$ by adjusting bias voltage of the polarization modulator, and this condition is not easy to implement in an experiment.

In this paper, we propose a two-tap complex-coefficient MPF using a polarizationdependent dual-parallel Mach-Zehnder modulator (PD-DPMZM). Compared with the

\footnotetext{
${ }^{*}$ Corresponding author: Pengge Ma; E-mail: mapenge@163.com.
} 
existing schemes, the proposed system requires only one optical source and one modulator. And the system structure is not complicated and extendable to more taps without adding additional laser source. By simply controlling a polarization controller (PC), the MPF is continually tunable in the full FSR without changing the filter shape and FSR.

\section{Principle}

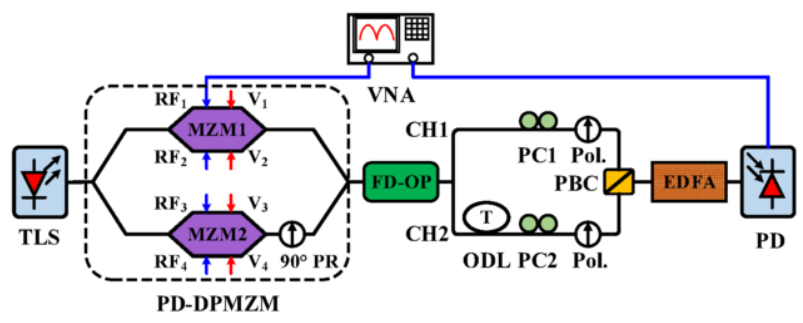

Figure 1. The schematic diagram of the proposed tunable MPF.

Figure 1 shows the proposed MPF. An optical carrier from a tunable laser source (TLS) is fed into a PD-DPMZM. A radio frequency (RF) signal generated by a vector network analyzer (VNA) is applied to one arm of the child MZM1 to accomplish a carriersuppressed dual-sideband (CS-DSB) modulation. The Child MZM2 has no RF signal and is biased at its maximum transmission point. Then the modulated signal is input to a Fourier-domain optical processor (FD-OP) to separate the upper sideband into the upper path and the lower sideband into the lower path. By doing this, an orthogonally polarized OSSB signal can be obtained at each path. Considering small signal modulation only, the output optical fields at the upper path and lower path are respectively given by

$$
\begin{aligned}
& E_{\text {upper }}(t)=\left[\begin{array}{c}
E_{x 1} \\
E_{y 1}
\end{array}\right] \propto \frac{1}{4} E_{0}\left[\begin{array}{c}
J_{1}(\beta) e^{j\left(\omega_{0}+\omega_{m}\right) t+j \frac{\pi}{2}} \\
e^{j \omega_{0} t}
\end{array}\right] \\
& E_{\text {lower }}(t)=\left[\begin{array}{c}
E_{x 2} \\
E_{y 2}
\end{array}\right] \propto \frac{1}{4} E_{0}\left[\begin{array}{c}
J_{1}(\beta) e^{j\left(\omega_{0}-\omega_{m}\right) t+j \frac{\pi}{2}} \\
e^{j \omega_{0} t}
\end{array}\right]
\end{aligned}
$$

Where $E_{0}$ is the amplitude of the optical carrier; $\omega_{0}$ and $\omega_{m}$ are the angular frequency of the optical carrier and RF signal, respectively; $J_{1}(\beta)$ is the first-order Bessel function of the first kind.

Two PCs are used to tune the phase difference between $E_{x}$ and $E_{y}$ in the two paths, respectively. The two OSSB signals are then polarization-combined by the polarizers (Pol.), respectively. If the combined signals are injected into a photodetector (PD), two electrical currents can be obtained 


$$
\begin{aligned}
& i_{\text {upper }}(t) \propto \sin \left(2 \alpha_{1}\right) \cos \left(\omega_{m} t+\phi_{1}+\frac{\pi}{2}\right) \\
& i_{\text {lower }}(t) \propto \sin \left(2 \alpha_{2}\right) \cos \left(\omega_{m} t-\phi_{2}-\frac{\pi}{2}\right)
\end{aligned}
$$

Where $\phi_{1}$ and $\phi_{2}$ are the phase differences between $E_{x}$ and $E_{y}$ in the two path, respectively; $\alpha_{1}$ and $\alpha_{2}$ are the polarization angles between the principal axis of the PD-DPMZM and the upper and lower polarizer, respectively. From Eq. (2), the two recovered RF signals can realize full $360^{\circ}$ phase shift by adjusting $\phi_{1}$ and $\phi_{2}$.

The signals after FD-OP propagate through different time delays by introducing an optical delay line (ODL) between the two optical paths. The recovered RF signal after PD is a summation of $i_{\text {upper }}(t)$ and $i_{\text {lower }}(t)$, as

$$
i(t)=i_{\text {upper }}(t)+i_{\text {lower }}(t)=\sum_{n=1}^{2} a_{n} \cos \left[\omega_{m} t+\phi_{n}+\omega_{m}(n-1) T\right]
$$

Where $a_{n}$ is the RF power in each tap; $\phi_{n}$ is the RF phase shift in each tap, which can be independently adjusted from 0 to $2 \pi$ by tuning the $\mathrm{PC}$ at each path; $\mathrm{T}$ is the relative time delay between the two taps.

The transfer function of the proposed MPF is given by

$$
H\left(\omega_{m}\right)=a_{1}+a_{2} e^{j \omega_{m} T} e^{j \Delta \phi}
$$

Where $e^{j \Delta \phi}$ is the complex coefficient, and $\Delta \phi$ is the phase difference between the two taps. The frequency response of the proposed MPF can be rewritten as

$$
H^{\prime}(f)=H(f+\Delta \phi / 2 \pi T)
$$

Where $f$ is the frequency of the RF signal.

In Eq. (5), the center frequency change of $\Delta \phi / 2 \pi T$ is realized by adjusting the phase difference between the two taps. The proposed MPF is continually tunable over the full FSR, since the phase shift covers full $360^{\circ}$ range.

\section{Experimental Results and Discussion}

A proof-of-concept experiment is implemented based on the setup shown in Figure 1. A $10.0 \mathrm{dBm}$ optical carrier at $\sim 1550.0 \mathrm{~nm}$ emitting from a TLS (Coherent Solutions MTP1000) is fed to the PD-DPMZM (Fujitsu FTM7980EDA). A -25 dBm RF signal generated by the VNA (Agilent 8722ES) is amplified by a broadband electrical amplifier (OA4MVM3), which has a wide frequency range from $30 \mathrm{kHz}$ to $45 \mathrm{GHz}$ with a gain of $27 \mathrm{~dB}$. The amplified RF signal is applied to the upper arm of child 
MZM1 of PD-DPMZM, and MZM1 is biased at its MITP to realize CS-DSB modulation. Child MZM2 is biased at its MATP without adding a RF signal. The modulated signal is sent to a FD-OP (Finisar Waveshaper 4000s). An orthogonally polarized OSSB signal is obtained at each path.

We measure the phase shift property of the recovered RF signal in the upper path while the lower path is off. The orthogonally polarized optical carrier and sideband are combined by the PBC and then amplified by an Erbium Doped Fiber Amplifier (EDFA, Conquer KG-EDFA-P) before sending to a PD (Optilab PD30). The output power of the EDFA is $1.3 \mathrm{dBm}$. The power-frequency response and phase-frequency response of the recovered RF signal within $8-26 \mathrm{GHz}$ are measured by the VNA. The $\mathrm{RF}$ phase shifts are recorded from $-180^{\circ}$ to $180^{\circ}$ with a step of $45^{\circ}$ by tuning $\mathrm{PC} 1$, as shown in Figure 2(a). It indicates that a continuously tunable phase shifter with stable performance over a wide bandwidth is obtained. Figure 2(b) shows that the relative power variations are within $\pm 2 \mathrm{~dB}$ for different phase shifts.
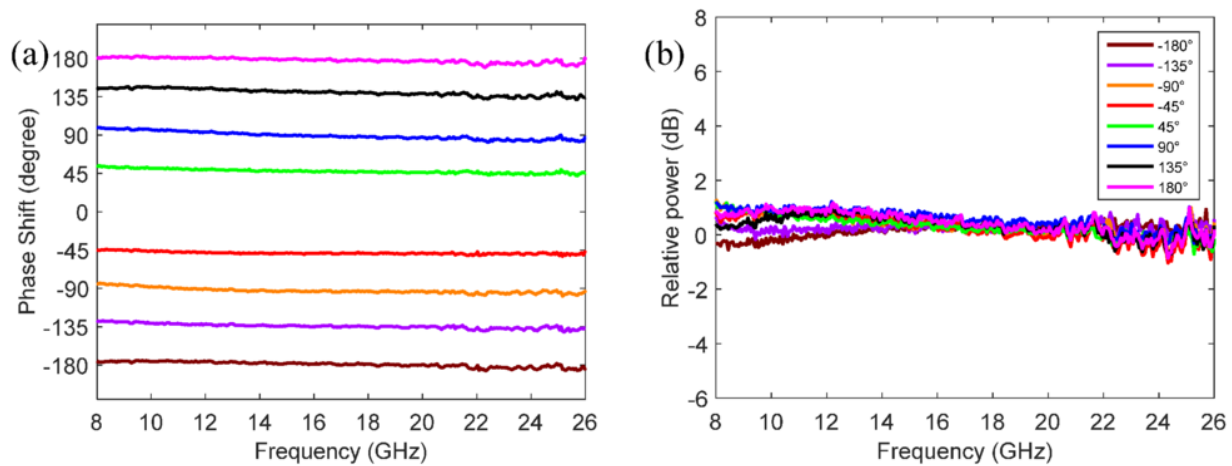

Figure 2. (a) Phase shifts of the RF signal within 8-26 GHz, (b) Relative power variations for different phase shifts.

In order to implement a two-tap MPF, an ODL is inserted into the lower path. The two orthogonally polarized OSSB signals in the two paths go through different time delays and then mutually interfere in the PD after the EDFA. Figure 3 shows the frequency responses of proposed filter with different center frequency within 20-21 GHz. As shown in figure 3(a), the FSR is maintained at $185 \mathrm{MHz}$ which is close to the theoretical value of $187 \mathrm{MHz}$ when the relative time delay $\mathrm{T}$ is about $5.35 \mathrm{~ns}$.
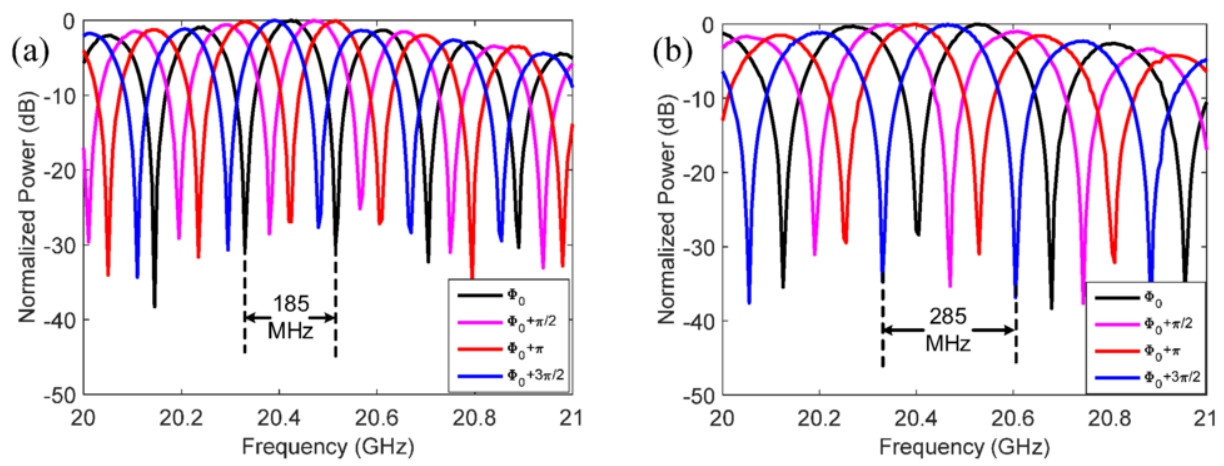

Figure 3. Measured frequency responses of the proposed MPF with FSRs of (a) $185 \mathrm{MHz}$ and (b) $285 \mathrm{MHz}$. 
To demonstrate the tunability of the FSR, different ODLs are used in the lower path to introduce different time delays between the two paths. When the relative time delay is changed to about $3.54 \mathrm{~ns}$, the measured FSR of the filter is nearly $285 \mathrm{MHz}$, as shown in Figure 3(b), which is approach to the theoretical value of $282 \mathrm{MHz}$. From Figure 3, the proposed MPF is tunable over the full FSR without affecting the FSR. Meanwhile, by inserting different ODL between the two paths, the FSR can be changed.

\section{Conclusions}

We propose and demonstrate a complex-coefficient MPF based on orthogonally polarized optical single-sideband modulation. The proposed MPF is continuously tunable in the full FSR without affecting the filter shape and FSR. The proposed MPF is simple, easily tunable, and extendable to more taps.

\section{Acknowledgments}

This work is supported in part by the National Natural Science Foundation of China (Nos. U1833203); and in part by the Key Scientific and Technological Projects in Henan Province (Nos. 212102210519).

\section{References}

[1] Minasian R A, Chan E, Yi X. Microwave photonic signal processing. Optics Express, 2013, 21(19):22918-22936.

[2] Sagues M, Loayssa A, Capmany J, et al. Tunable complex-coefficient incoherent microwave photonic filters based on optical single-sideband modulation and narrow-band optical filtering. Optical Fiber Communication Conference. 2007: OWU5.

[3] Wang W T, Liu J G, Mei H K, et al. Microwave photonic filter with complex coefficient based on optical carrier phase shift utilizing two stimulated Brillouin scattering pumps. IEEE Photonics Journal, 2015, $7(1): 1-8$.

[4] Xu E, Yao J. Frequency- and notch-depth-tunable single-notch microwave photonic filter. IEEE Photonics Technology Letters, 2015, 27(19): 2063-2066.

[5] Xu D, Cao Y, Tong Z, et al. A continuously tunable microwave photonic notch filter with complex coefficient based on phase modulation. Optoelectronics Letters, 2017, 13(1): 13-15.

[6] Zhang C, Yan L S, Pan W, et al. A tunable microwave photonic filter with a complex coefficient based on polarization modulation. IEEE Photonics Journal, 2013, 5(5):5501606-5501606. 